Rev Biomed 2002; 13:84-92.

\title{
Clínica del asma: ¿en realidad es útil?
}

\author{
Artículo Original
}

Eduardo A. Lara-Pérez.

Hospital de Gineco-Pediatría 71, Instituto Mexicano del Seguro Social, Veracruz, Veracruz, México.

\section{RESUMEN.}

Introducción. Los programas del asma, no se cumplen, por falta de información y capacitación. El objetivo de este trabajo fue comparar el beneficio de manera objetiva de la "Clínica del Asma".

Material y métodos. Grupo I: asisten; Grupo II: no asisten. Se comparó FEM (flujo espiratorio máximo) en crisis e intercrisis y terapia ambulatoria.

Resultados. 64 niños por grupo. Grupo I: diferencia ingreso/egreso (valores medios de FEM) 60 L/min. Grupo II: diferencia ingreso/ egreso $87.5 \mathrm{~L} / \mathrm{min}$. Intercrisis: Grupo I: 87.5 L/min FEM, 66.04\% valores promedio a 10 normal. Grupo II; $102.5 \mathrm{~L} / \mathrm{min}$ con $71.25 \%$ promedio FEM. Al clasificar la gravedad no hubo diferencias significativas. Grupo I: medicamentos $95.31 \%$, Grupo II; $51.56 \%$.

Discusión. El FEM en los programas para el asma es básico, pero en la practica no se utiliza, sin objetividad no se puede evaluar el beneficio de la "Clínica del Asma" y la terapia ambulatoria o en la crisis. Hay falta de información y capacitación del uso correcto del FEM en ambos grupos. Los valores medios del FEM son mejores en crisis e intercrisis y menos medicamentos en los asmáticos que no asisten a la clínica del asma. Debe favorecerse la aplicación de los programas del asma y promover la coparticipación familiar.

(Rev Biomed 2002; 13:84-92)

Palabras clave: Asma, flujo espiratorio máximo, crisis asmáticas.

\section{SUMMARY.}

Asthma clinic: is it really useful?

Introduction. Due to the lack of information and training asthma programs are not being carried out.

The objective of this work is compare the benefits of the "Asthma clinic".

Material and methods. Group I: patients attend the clinic. Group II: patients don't

Solicitud de sobretiros: Dr. Eduardo A. Lara-Pérez, Pedro de Alvarado 201, Frac. Reforma, C.P. 91919, Veracruz, Ver., México. 


\section{EA Lara-Pérez.}

attend the clinic. Maximun expiratory flow (MEF) was compared during crisis, inter-crisis and ambulatory therapy.

Results. 64 children per group; Group I admission/discharge average values of MEF 60 $\mathrm{L} / \mathrm{min}$. Group II admission/discharge difference was $87.5 \mathrm{~L} / \mathrm{min}$. Intercrisis: Group I had $87.5 \mathrm{~L} / \mathrm{min} \mathrm{MEF}, 66.04 \%$ showed values average to normal. Group II $102.5 \mathrm{~L} / \mathrm{min}$ with $71.25 \%$ average MEF. There were no serious differences on classifying the seriousness of the disease. Group I: $95.31 \%$ took medicine while $51.56 \%$ of group II.

Discussion. The MEF is basic to any asthma program, but in practice it isn't used, without objectivity it is impossible to evaluate the benefits of the asthma clinic and the ambulatory therapy or during crisis. There is a lack of information and training of the correct use of the MEF in both groups. The average values of MEF are better in crisis and intercrisis and less medicine is taken by patients who don't attend the asthma clinic, which should favour the application of asthma programs and promote the participation of the family.

(Rev Biomed 2002; 13:84-92)

Key words: Asthma, maximum expiratory flow, asthmatic crisis.

\section{INTRODUCCIÓN.}

Los principales problemas de salud de países en vías de desarrollo son las enfermedades crónicas no transmisibles y dentro de éstas el asma bronquial ocupa, un lugar importante (1-4). Desde hace décadas la preocupación de médicos e instituciones por unificar criterios en el diagnóstico y tratamiento del asma infantil, dieron como consecuencia la creación de las "Clínicas del Asma" como una respuesta e intento por estandarizar la atención a este padecimiento.
Hace años se elaboró un programa internacional para la atención del asma llamado "GINA" (Global initiative of asthma) por sus siglas en ingles, el cual ha basado sus criterios de clasificación, manejo y control con signos o síntomas y pruebas de la función respiratoria, específicamente en el Flujo Espiratorio Máximo (FEM) y el Volumen Espiratorio Forzado del primer segundo (FEV1) (5).

A pesar de la aceptación del "GINA" por la mayoría de los países en el mundo, en la realidad este programa no se lleva cabalmente, sobre todo por la falta de aplicación de los parámetros objetivos; específicamente el FEM y más difícilmente de espirometría, ni en las crisis, ni en los periodos asintomáticos o de intercrisis, a pesar que se ha demostrado que los asmáticos tienen FEM menor al esperado (6) comparándolo con los valores de FEM en niños sanos (7).

La falta de información, capacitación y orientación del paciente en algunas "Clínicas del Asma" se nota por el desconocimiento del FEM y sus beneficios así como la ausencia de coparticipación de padres y familiares en el manejo de la enfermedad (8-10). Los asmáticos sometidos a un programa integral y comprometido con sus familiares; disminuyen el número de crisis, el consumo de medicamentos para controlar sus crisis y la terapéutica de control ambulatorio o intercrisis, sin necesidad de ingresos hospitalarios, resultados encontrados en Estados Unidos (11) y en población hispana en Europa (12).

La Sociedad Latinoamericana de Alergia e Inmunología y el Instituto Nacional de Corazón, Pulmón y Sangre del Servicio de Salud Pública de los Estados Unidos (13) avalan lo anterior e incluyen el uso de inhaladores, medidores de flujo (flujómetros), recomendaciones para el control ambiental, automanejo de algunos medicamentos y el apoyo que el asmático debe recibir de sus

\section{Revista Biomédica}


Utilidad de la Clínica del Asma.

familiares, con el propósito de mejorar la calidad de su vida. Desgraciadamente existe gran resistencia para el uso del FEM, especialmente en los médicos, a pesar de las ventajas que este tiene para predecir nuevas crisis $(14,15)$.

Por esta razón, se impone la evaluación sistemática y objetiva de la calidad de los servicios de las "Clínicas del Asma", con un enfoque multidisciplinario y con el conocimiento de que la evaluación implica dimensionar lo que existe y compararlo con lo que desearíamos o debiéramos encontrar e investigar de qué manera podríamos acortar las eventuales distancias entre realidad, deseo u obligación $(16,17)$.

Con este trabajo intentamos comparar de manera objetiva el beneficio en orientación, información, capacitación y terapia, con un plan o programa para el niño asmático que asiste a la "Clínica del Asma" de los que no asisten y sólo reciben de manera irregular sin planeación, información y manejo adecuado para su padecimiento.

\section{MATERIAL Y MÉTODOS.}

En el Hospital No. 71 del Instituto Mexicano del Seguro Social (IMSS) de la ciudad y puerto de Veracruz, se aplicó de manera prospectiva, longitudinal y observacional, en urgencias e inhaloterapia, un cuestionario a pacientes pediátricos de 5 a 16 , años que acudieron a urgencias por presentar crisis asmática, en el periodo del 20 de agosto de 1999 al 20 de noviembre de 2000. Fueron excluidos del estudio los niños que no completaron su cuestionario. El cuestionario contenía los siguientes datos: nombre, edad, género, numero de filiación, talla, medicación o terapia de manera regular y por más de un mes previa a la crisis, asistencia a la clínica del asma o no; se preguntó si conocían o se habían tomado previamente el FEM, aunque de cualquier manera a todos los niños se les informó y capacitó acerca de la flujometria. Se tomó el FEM al ingreso y egreso de urgencias y posteriormente dentro de los 15 días subsecuentes a su crisis en condiciones asintomáticas de manifestaciones respiratorias.

\section{Medición y clasificación de las variables.}

El cuestionario fue llenado por el mismo investigador. Para la medición de talla; se utilizó una bascula con estandímetro marca Detecto ${ }^{\circledR}$.

Para considerar la medicación o terapia ambulatoria o de intercrisis esta debió tomarse regularmente durante un mínimo de un mes. A pesar de no ser la inmunoterapia un medicamento, se consideró como tratamiento de la intercrisis, por estar específicamente indicado como terapia del asma.

Para la medición del FEM se utilizó el Flujómetro de Assess ${ }^{\circledR}$ previa información de la metodología para su aplicación: no haber realizado ejercicio físico cuando menos media hora antes, no haber ingerido bebidas calientes o estimulantes y en nuestra experiencia tomamos el valor más alto de 5 intentos (tratando con ello; evitar el error de aprendizaje). Para la clasificación de las crisis asmáticas por flujometria se utilizo el semáforo de Mendoza (18) o sea de la siguiente manera: normal 91 a $100 \%$, leve con valor de 71 a $90 \%$, moderado del 50 a $70 \%$ y grave con menos del $50 \%$ de FEM de acuerdo al mejor valor de FEM estando asintomático.

Para clasificar las condiciones del FEM en el periodo asintomático se clasifico de acuerdo a lo recomendado por Lara-Pérez (6). Esto es: normal 91 a $100 \%$, leve 71 a $90 \%$, moderado 51 a $70 \%$ y grave $50 \%$ o menos del FEM de escolares sanos (7) cuyas fórmulas son como siguen: para masculinos $6.10 \mathrm{x}$ talla en $\mathrm{cm}-$ $500.71=$ FEM y femeninos; $6.17 \mathrm{x}$ talla en $\mathrm{cm}$ 


\section{EA Lara-Pérez.}

$-523.19=$ FEM

\section{Captura, método y análisis estadístico.}

Para hacer dos grupos comparables, se ajustaron los grupos: los que asisten con regularidad a la Clínica del Asma (GRUPO I) por cuando menos 3 meses y los que no asisten a la Clínica del Asma (GRUPO II). Se procuró que fueran lo más similares posible, tanto en número, talla y sexo, para su mejor comparación.

En ambos grupos se determinaron; valores mínimos, máximos, medianas, y desviación estándar de edad, FEM (tanto al ingreso, egreso y asintomático). Se valoró mejoría con la diferencia de las medianas del FEM ingreso/ egreso y para los periodos de asintomáticos o sin crisis, comparando los valores del FEM con porcentajes promedios comparados con lo normal.

La variable de medicamentos o terapia se hizo de manera nominal y porcentual, comparando resultados por grupos.

\section{RESULTADOS.}

Se seleccionaron 128 niños asmáticos en dos grupos, cada grupo con 64 casos. El grupo que asiste a la Clínica del Asma o GRUPO I, con 36 masculinos $(56.25 \%)$ y 28 femeninos (43.75\%), la edad mínima de 4 años 7 meses y la máxima de 14 años (mediana de 9 años 2 meses, desviación estándar de 79.9 meses). Los niños que no asisten a la Clínica del Asma o GRUPO II, con 40 masculinos (62.5\%), y 24 femeninos $(37.5 \%)$, la edad mínima de 5 años y la máxima de 15 años (mediana de 10 años con desviación estándar de 84.8 meses).

La talla para el GRUPO I fue mínima de $110 \mathrm{~cm}$, máxima de $165 \mathrm{~cm}$ y mediana de 138 cm (desviación estándar de $38.18 \mathrm{~cm}$ ). En el GRUPO II la talla mínima fue de $110 \mathrm{~cm}$ y la máxima de $164 \mathrm{~cm}$ y mediana de $139.5 \mathrm{~cm}$ con desviación estándar de $34.6 \mathrm{~cm}$.

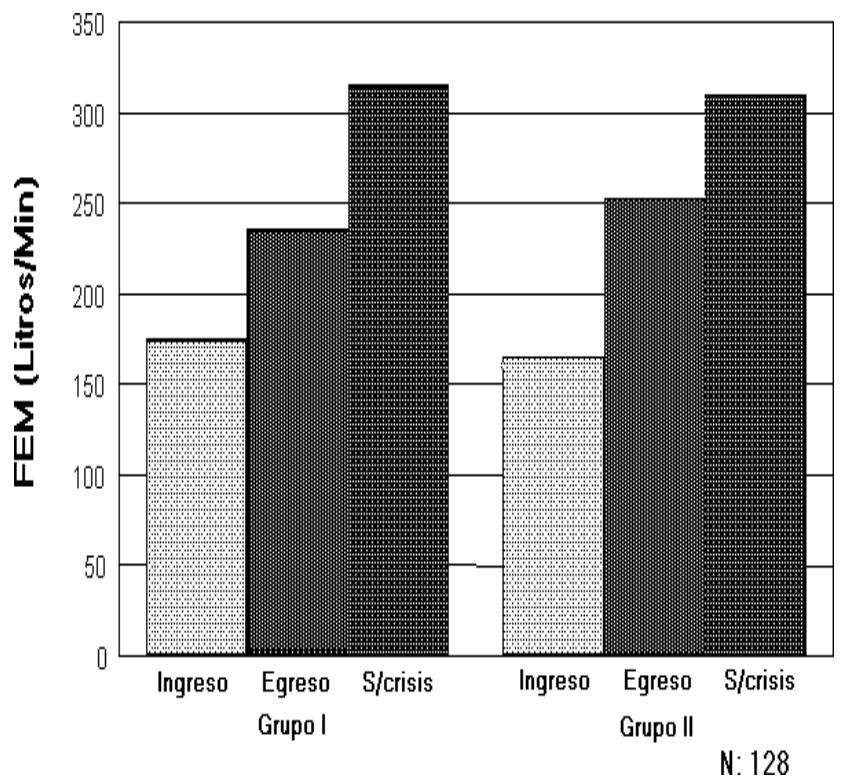

Figura 1.- Comportamiento de los valores medios de FEM (Flujo espiratorio máximo) en ambos grupos. Al ingreso y egreso de urgencias (crisis), y sin crisis (asintomático)

Ningún grupo tenia conocimiento del FEM ni costumbre de su uso como medida de control.

FEM al ingreso: GRUPO I; mínimo 40

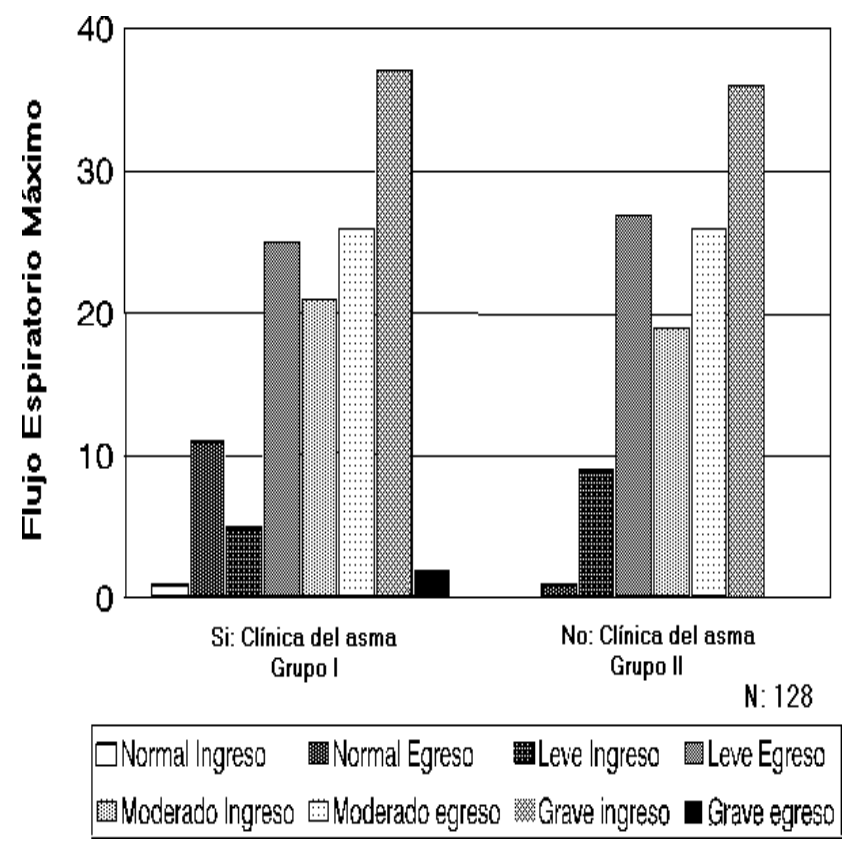

Figura 2.- Clasificación de las crisis asmáticas por FEM (Flujo espiratorio máximo) al ingreso y egreso en ambos grupos.

\section{Revista Biomédica}




\section{Utilidad de la Clínica del Asma.}

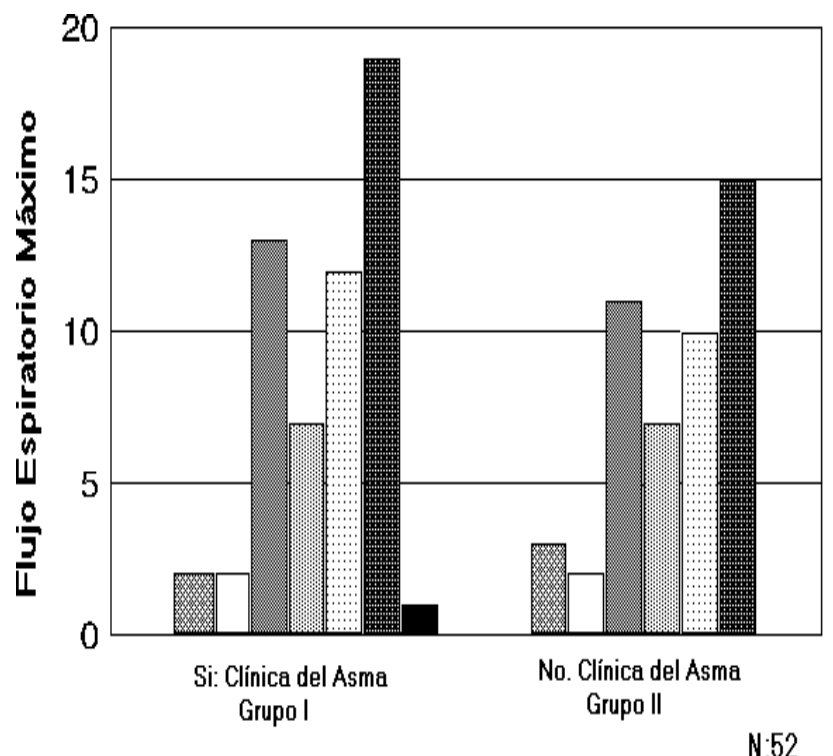

N:52

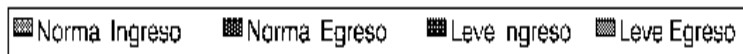

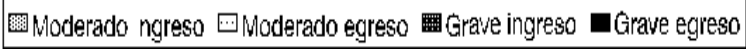

Figura 3.- Clasificación de las crisis asmáticas en los Femeninos; por FEM (Flujo espiratorio máximo) al ingreso y egreso en ambos grupos.

$\mathrm{L} /$ min, máximo $300 \mathrm{~L} / \mathrm{min}$. y mediana de 175 $\mathrm{L} / \mathrm{min}$ con desviación estándar de 35.3. Para el GRUPO II FEM al ingreso fue mínimo 40 $\mathrm{L} / \mathrm{min}$, máximo $340 \mathrm{~L} / \mathrm{min}$ y mediana de 139.5 L/min (desviación estándar de 21.21).

FEM al egreso: GRUPO I, mínimo $50 \mathrm{~L} /$ min, máximo $410 \mathrm{~L} / \mathrm{min}$ y mediana de $235 \mathrm{~L} /$ min (desviación estándar de 77.78). Para el GRUPO II FEM al egreso fue mínimo $60 \mathrm{~L} /$ min, máximo $345 \mathrm{~L} / \mathrm{min}$ y mediana de 252.5 L/min (desviación estándar de 10.60).

FEM asintomático: GRUPO I; mínimo $100 \mathrm{~L} / \mathrm{min}$, máximo $450 \mathrm{~L} / \mathrm{min}$ y mediana de $315 \mathrm{~L} / \mathrm{min}$ (desviación estándar de 190.91). Para el GRUPO II FEM asintomático mínimo $100 \mathrm{~L} / \mathrm{min}$, máximo $420 \mathrm{~L} / \mathrm{min}$ y mediana de $310 \mathrm{~L} / \mathrm{min}$ (desviación estándar de 127.27) (figura 1).

La diferencia ingreso/egreso del FEM durante la crisis para el Grupo I de $60 \mathrm{~L} / \mathrm{min}$ a diferencia del Grupo II que fue de $87.5 \mathrm{~L} /$ min. Para el periodo de intercrisis los valores

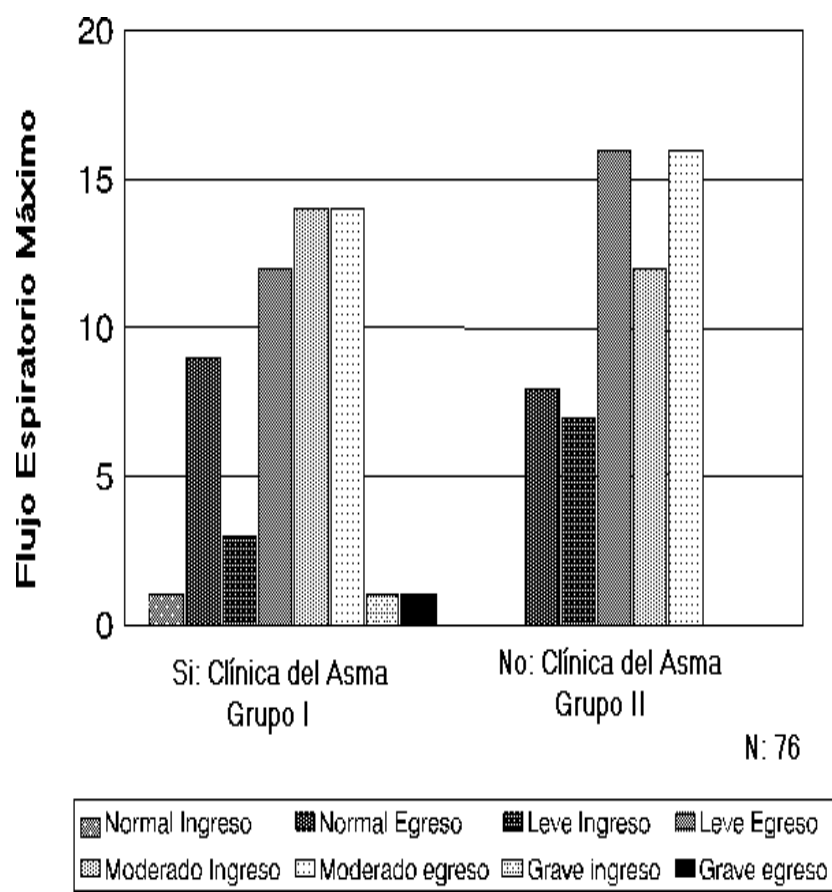

Figura 4.- Clasificación de las crisis asmáticas en los Masculinos; por FEM (Flujo espiratorio máximo) al ingreso y egreso en ambos grupos.

medios de FEM Grupo I 87.5 contra 102.5 del Grupo II con una diferencia de $15 \mathrm{~L} / \mathrm{min}$ a favor del Grupo II. Igualmente el porcentaje de valores medios de FEM comparado a lo esperado de valores de sanos fue para el Grupo I de $66.04 \%$, a diferencia del Grupo II con $71.25 \%$, lo que resulta $5.21 \%$ mejor para el Grupo II.

Al comparar gravedad de las crisis de acuerdo al semáforo de Mendoza (18) resultó lo siguiente (figura 2):

Al ingreso: GRUPO I normal 1 caso (1.56\%), leve 5 casos (7.81\%), moderado 21 casos $(32.81 \%)$, y grave 37 casos $(57.81 \%)$.

GRUPO II normal 0 casos, leve 9 casos (14.06\%), moderado 19 casos (29.68\%), y grave 36 casos $(56.25 \%)$.

Al egreso: GRUPO I normal 11 casos (17.18\%), leve 25 casos (39.06\%), moderado 26 casos $(40.62 \%)$, y grave 2 casos $(3.12 \%)$.

GRUPO II; normal 11 casos (17.18\%), leve 27 casos $(42.18 \%)$, moderado 26 casos 
EA Lara-Pérez.

(40.62\%), y grave 0 casos.

Clasificación de gravedad por género:

Al ingreso: GRUPO I femeninos; normal 0 casos, leve 2 casos $(7.14 \%)$, moderado 7 casos (25\%), y grave 19 casos $(67.85 \%)$.

GRUPO II femeninos: normal 0 casos, leve 2 casos $(8.33 \%)$, moderado 7 casos $(29.16 \%)$, y grave 15 casos $(62.5 \%)$.

Al egreso: GRUPO I femeninos; normal 2 casos $(7.14 \%)$, leve 13 casos (46.42\%), moderado 12 casos $(42.85 \%)$, y grave 1 caso (3.57\%).

GRUPO II femeninos; normal 3 casos (12.5\%), leve 11 casos (45.83\%), moderado 10 casos $(41.66 \%)$, y grave 0 casos figura 3 .

Al ingreso GRUPO I masculinos: normal 1 caso $(2.77 \%)$, leve 3 casos $(8.33$ $\%)$, moderado 14 casos $(38.88 \%)$, y grave 18 $\operatorname{casos}(50 \%)$.

GRUPO II masculinos: normal 0 casos, leve 7 casos $(17.5 \%)$, moderado 12 casos (30\%), y grave 21 casos $(52.5 \%)$.

Al egreso; GRUPO I masculinos: normal 9 casos $(25 \%)$, leve 12 casos $(33.33 \%)$, moderado 14 casos $(38.88 \%)$, y grave 1 caso $(2.77 \%)$.

GRUPO II masculinos: normal 8 casos (20\%), leve 16 casos (40\%), moderado 16 casos $(40 \%)$, y grave 0 casos (figura 4$)$.

El FEM en periodo asintomático o intercrisis se encontró lo siguiente:

GRUPO I (uno y otro género); normal 23 casos $(35.93 \%)$, leve 26 casos $(40.62 \%)$, moderado 10 casos ( $15.62 \%)$, y grave 5 casos $(7.81 \%)$.

GRUPO II uno y otro género; normal 13 casos $(20.31 \%)$, leve 32 casos $(50 \%)$, moderado 18 casos $(28.12 \%)$, y grave 1 casos (1.56\%), (figura 5).

La medicación o terapia utilizada de manera ambulatoria o en la intercrisis se

Cuadro 1

\begin{tabular}{|c|c|c|c|c|c|}
\hline \multicolumn{3}{|c|}{$\begin{array}{c}\text { Grupo I } \\
\text { Si asistieron (Clínica del Asma) }\end{array}$} & \multicolumn{3}{|c|}{$\begin{array}{c}\text { Grupo II } \\
\text { No asistieron (Clínica del Asma) }\end{array}$} \\
\hline Terapia & No. & $\%$ & Terapia & No. & $\%$ \\
\hline Ninguna terapia & 3 & $4.68 \%$ & Ninguna terapia & 31 & $48.43 \%$ \\
\hline Con terapia & 61 & $95.31 \%$ & Con terapia & 33 & $51.56 \%$ \\
\hline Monoterapia & 22 & $34.42 \%$ & Monoterapia & 24 & $72.72 \%$ \\
\hline Terapia doble & 39 & $65.57 \%$ & Terapia doble & 9 & $27.27 \%$ \\
\hline Monoterapia & 22 & $100 \%$ & Monoterapia & 24 & $100 \%$ \\
\hline Astemizol & 1 & $4.54 \%$ & Astemizol & 0 & $0.00 \%$ \\
\hline Cromoglicato & 2 & $9.09 \%$ & Cromoglicato & 0 & $0.00 \%$ \\
\hline Ketotifeno & 4 & $18.18 \%$ & Ketotifeno & 4 & $16.66 \%$ \\
\hline Salbutamol & 7 & $31.81 \%$ & Salbutamol & 12 & $50.00 \%$ \\
\hline Inmunoterapia & 8 & $36.36 \%$ & Inmunoterapia & 8 & $33.33 \%$ \\
\hline Terapia doble & 78 & $100 \%$ & Terapia doble & 18 & $100 \%$ \\
\hline Beclometasona & 1 & $1.28 \%$ & Beclometasona & 1 & $5.55 \%$ \\
\hline Cromoglicato & 4 & $5.12 \%$ & Cromoglicato & 1 & $5.55 \%$ \\
\hline Inmunoterapia & 4 & $5.12 \%$ & Inmunoterapia & 1 & $5.55 \%$ \\
\hline Astemizol & 5 & $6.41 \%$ & Astemizol & 2 & $11.11 \%$ \\
\hline Ambroxol & 14 & $17.94 \%$ & Ambroxol & 4 & $22.22 \%$ \\
\hline Ketotifeno & 25 & $32.05 \%$ & Ketotifeno & 3 & $16.66 \%$ \\
\hline Salbutamol & 25 & $32.05 \%$ & Salbutamol & 6 & $33.33 \%$ \\
\hline
\end{tabular}


Utilidad de la Clínica del Asma.

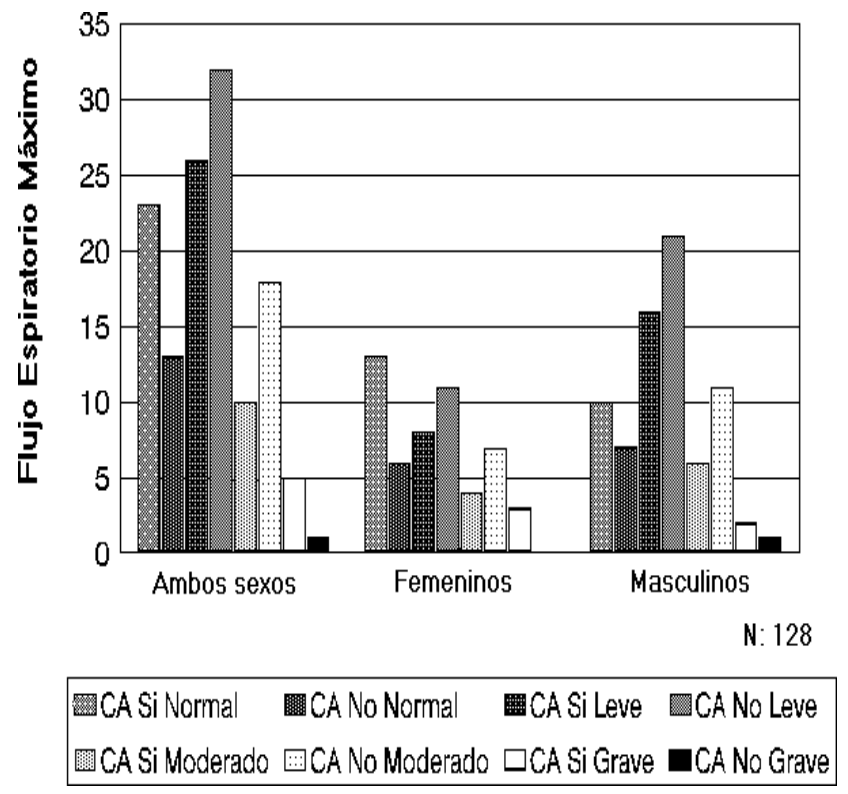

Figura 5.- Clasificación del FEM (Flujo espiratorio máximo) asintomáticos (sin crisis) en ambos grupos.

describe en el cuadro 1.

\section{DISCUSIÓN.}

Los resultados son mas favorables en el FEM y uso de medicamentos en niños que no asisten a la "Clínica del Asma" que los que asisten, lo que nos permite hacer algunas consideraciones. El FEM es la herramienta clínica de evaluación de la función pulmonar; practica, operativa, y fundamental en los consensos y guías de atención del asma infantil (5). Desgraciadamente la falta de información a pacientes para su uso, y la resistencia por parte de algunos médicos, hace que se desperdicien las ventajas para predecir nuevas crisis $(14,15)$, sobre todo si el control se realiza por semanas adecuando la terapéutica y disminuyendo riesgos (19). La alteración del FEM en las crisis disminuye entre 15 a $30 \%$ situación que podría prevenirse, al notarse deterioro progresivo del FEM, aunque hay opiniones de que el FEM no tiene utilidad diagnostica en el asma (20). Existen estudios que al comparar la población asmática con la sana el $77 \%$ tienen FEM anormalmente bajo (6).

Otro beneficio del FEM es la disminución de medicamentos ambulatorios y los ingresos a urgencias (21), es también es un excelente parámetro de evaluación terapéutica en los servicios de urgencia y evolución de la gravedad de la crisis $(18,22)$, resultando con ello una mejor calidad de atención (23). Sin embargo, es recomendable realizar una buena y persistente información con capacitación en la toma correcta del FEM para evitar errores de aplicación o de interpretación (24). Cuando se logre convencer a pacientes y médicos de estos beneficios, el control de este padecimiento se lograra a distancia, como se ha demostrado con programas de apoyo telefónico, disminuyendo los ingresos hospitalarios (25).

En el futuro cercano las "Clínicas del Asma" tendrán que incorporar rutinariamente del FEM promoviendo su uso en casa dentro del control ambulatorio y evaluación de las crisis, ya que el FEM es el mejor método objetivo de control del asma (26).

Llama la atención en este trabajo que ambos grupos utilicen medicamentos que no solo no están indicados en las intercrisis del asma, sino que por el contrario, están incluso contraindicados ya que no hay ningún beneficio con su uso (salbutamol y ambroxol) y sólo aumentan riesgos, gastos, ignorancia y no mejoran las condiciones del asmático, ni su satisfacción.

\section{REFERENCIAS.}

1.- Meza-Morales A. Uso de esteroides en niños asmáticos. Rev Alerg Mex 1995; 4:74-77.

2.- Cuba. Ministerio de Salud Pública. Objetivos, propósitos y directrices para incrementar la salud de la población cubana 1992-2000. La Habana: Editorial Ciencias Médicas:1992.

3.- Cuba. Ministerio de Salud Pública. Informe anual 


\section{EA Lara-Pérez.}

de datos estadísticos. Cuba 1996.

4.- Rodríguez de la Vega A. Investigación sobre la prevalencia de asma bronquial en Cuba. Rev Cubana Adm Salud 1983; 9:95-118.

5.- British Thoracic Society. The British guidelines on asthma management. 1995 review and position statement. Thorax 1997; 52 (Suppl. 1): S1-S21.

6.- Lara-Pérez EA. Flujo espiratorio máximo en asmáticos asintomáticos. Archiv Invest Ped Mex. 2000; 3: 343-8.

7.- Lara-Pérez EA. Flujo espiratorio máximo en escolares sanos. Archiv Invest Ped Mex 1999; 2:16570 .

8.- Donaghy D. The asthma specialist and patient education. Prof Nurse 1995; 11:160-2.

9.- Salmún N, Kohan M. Fundaler y la educación del asmático y su familia. Arch Argent Alergia Inmunol Clin 1994; 25:254-8.

10.- Arias-Díaz A, Pernas-Gómez M, Martín G. Aplicación de un programa de entrenamiento para el automanejo del asma bronquial Rev Cubana Med Gen Integr 1998; 14:335-9.

11.- Kotses H. A self management program for adult asthma part I: development evaluation. J Aller Clin Inmunol 1995; 95:259-70.

12.-Ignacio García JM, González Santos P. Asthma self/management education program by name monitoring of peak expiratory flow. Am J Respir Crit Care Med 1995; 195:353-9.

13.- Department of Health and Human Services International Consensus. Report and Diagnosis and Management of Asthma, 1992.

14.- Mendenhall AB, Tsien AY. Evaluation of physician and patient compliance with the use of peak flow meters in commercial insurance and Oregon health plan asthmatic populations. Ann Allergy Asthma Immunol 2000; 84:523-7.

15.- Boggs PB, Wheeler D, Washburne WF, Hayati F. Peak expiratory flow rate control chart in asthma care: chart construction and use in asthma care. Ann Allergy Asthma Immunol 1998; 81:552-62.

16.- Boland M. Lo que esperan los pacientes de los médicos. Foro Mundial de la Salud 1995; 16:251-5.

17.- Nutting P. Métodos de evaluación de la calidad en atención primaria. Guías para clínicos. Barcelona: Ediciones S.G; 1991. p. 114.

18.- Mendoza GR. Peak flow monitoring at home: an interactive process between you and your physician. En : Spector A, editor. Understanding asthma. Palatine III: American College of Allergy and Immunology; 1989. p. 155-62.

19.- Alcock SM; Mamun M; Prescott RJ; Connolly CK. Symptoms and pulmonary function in asthma. Respir Med, 1998; 92:849-57.

20.- Thiadens HA, De Bock GH, Van Houwelingen JC, Dekker FW, De Waal MW, Springer MP, Postma DS. Can peak expiratory flow measurements reliably identify the presence of airway obstruction and bronchodilator response as assessed by FEV(1) in primary care patients presenting with a persistent cough? . Thorax 1999; 54:1055-60.

21.- Emond SD, Woodruff PG, Lee EY, Singh AK, Camargo CA Jr . Effect of an emergency department asthma program on acute asthma care. Ann Emerg Med 1999; 34:321-5.

22.- McCarren M, Zalenski RJ, McDermott M, Kaur $\mathrm{K}$. Predicting recovery from acute asthma in an emergency diagnostic and treatment unit. Acad Emerg Med 2000; 7:28-35.

23.- Abisheganaden J, Ng SB, Lam KN, Lim TK. Peak expiratory flow rate guided protocol did not improve outcome in emergency room asthma. Singapore Med J. 1998; 39:479-84.

24.- Nolan S, Tolley E, Leeper K, Strayhorn Smith $\mathrm{V}$, Self T. Peak expiratory flow associated with change in positioning of the instrument: comparison of five peak flow meters. J Asthma 1999; 36:291-4.

25.- Kokubu F; Nakajima S; Ito K; Makino S; Kitamura S; Fukuchi Y; Mano K; Sano Y; Inoue H; Morita Y; Fukuda K; Akiyama K; Adachi M;

\section{Revista Biomédica}


Miyamoto T . Hospitalization reduction by an asthma tele-medicine system. Arerugi 2000; 49:19-31.

26.- Ueshima Y, Kikuchi T, Kaneko N, Kaneshige H, Suzuki H. Study of how to continue peak flow monitoring for patients with bronchial asthma: questionnaire to continuation and interruption patients. Arerugi 2000; 49:420-7. 